\title{
Laser-Assisted Bending of Sharp Angles With Small Fillet Radius on Stainless Steel Sheets: Analysis of Experimental Set-Up and Processing Parameters
}

\author{
Annamaria Gisario • Massimiliano Barletta • \\ Simone Venettacci • Francesco Veniali
}

Accepted: 15 January 2015 / Published online: 29 January 2015

(C) Springer New York 2015

\begin{abstract}
Achievement of sharp bending angles with small fillet radius on stainless steel sheets by mechanical bending requires sophisticated bending device and troublesome operational procedures, which can involve expensive molds, huge presses and large loads. In addition, springback is always difficult to control, thus often leading to final parts with limited precision and accuracy. In contrast, laserassisted bending of metals is an emerging technology, as it often allows to perform difficult and multifaceted manufacturing tasks with relatively small efforts. In the present work, laser-assisted bending of stainless steel sheets to achieve sharp angles is thus investigated. First, bending trials were performed by combining laser irradiation with an auxiliary bending device triggered by a pneumatic actuator and based on kinematic of deformable quadrilaterals. Second, laser operational parameters, that is, scanning speed, power and number of passes, were varied to identify the most suitable processing settings. Bending angles and fillet radii were measured by coordinate measurement machine. Experimental data were elaborated by combined ANalysis Of Mean (ANOM) and ANalysis Of VAriance (ANOVA). Based on experimental findings, the best strategy to achieve an aircraft prototype from a stainless steel sheet was designed and implemented.
\end{abstract}

Keywords Laser-assisted bending $\cdot$ Sharp angles $\cdot$ Bending device $\cdot$ Dimensional accuracy $\cdot$ Precision

\footnotetext{
A. Gisario $\cdot$ S. Venettacci $\cdot$ F. Veniali

Dipartimento di Ingegneria Meccanica ed Aerospaziale, Via Eudossiana, 18, 000184 Rome, Italy

M. Barletta ( $\triangle)$

Dipartimento di Ingegneria dell'Impresa, Via del Politecnico, 1, 00133 Rome, Italy

e-mail: barletta@ing.uniroma2.it
} 


\section{Introduction}

Achievement of sharp bending angles with small fillet radius on stainless steel sheets by mechanical bending requires sophisticated bending device and troublesome operational procedures, which can involve expensive molds, huge presses and large loads. In addition, springback is always difficult to control, thus often leading to final parts with limited precision and accuracy. In contrast, laser-assisted bending of metals is an emerging technology, as it often allows to perform difficult and multifaceted manufacturing tasks with relatively small efforts. During mechanical shaping, workpiece stores a lot of energy in the elastic field, which is then released once the external load is removed. This can cause process inaccuracy and significant deviations of the final parts from the expected targets $[13,4,6,14]$. Accuracy of shaping process is thus dependent on the extent of workpiece to store energy in the elastic field during bending. Similarly, the extent of stored energy is dependent on workpiece material (especially, elastic modulus, yield strength and hardening coefficient), workpiece-mold interactions (especially, frictions) and loading systems (especially, transient effect due to reverse loading or repeated loading). For this reason, control of springback is always extremely troublesome, being this a source of major unpredictability when processing metal blanks by mechanical shaping $[18,2]$. Advanced models based on materials constitutive equations could allow better results in springback prediction [20], while it would be a time and cost-consuming approach, not suitable on large scale or industrial applications.

Empirical methods rather than systematic knowledge is thus widely used in the practice to take into account springback in metal shaping. However, the stringent requirements of market for alternative and exotic alloys as well as the frequent recourse to innovative design make springback-related phenomenon greatly tangible and still more difficult to deal with. First pioneeristic studies to limit springback in metal shaping date back to late $80 \mathrm{~s}$, when troublesome control procedures, involving different steps of restraining force, were proposed in the literature [15]. However, the main drawbacks of these methods were related to their sensitivity to workpiece material (especially, friction) and their intrinsic unsuitability to face springback issues in difficult shaping operations. In mid 90s, closed-loop algorithms to control binder force and increase control and accuracy in shaping process were first proposed [23]. More recently, combination of hot dies and cold punches was proposed to reduce springback [17]. Nevertheless, temperature control in sophisticated shaping device is significantly time and cost-consuming, discouraging any further attempts. In the last decade, literature abounds with empirical [12], methodological [25] and theoretical [26] analyses to face springback-related issues. Alternative approaches include hybrid forming technologies in which laser is used to assist mechanical bending. Laser heating reduces typical loads necessary for metal shaping, thus intrinsically limiting the onset of stress field inside the workpiece and thus reducing springback. Laser-assisted bending has been attracting a lot of interest, with several studies being reported in the literature $[1,5$, $21]$ in which difficult to bend materials were successfully processed by hybrid techniques. More recently, additional laser sources, empirical methods and modelling analyses were investigated in shaping of metallic and non metallic sheets [7, 19]. However, limited availability of experimental data, poor knowledge of laser-material interaction during laser assisted bending, scarcity of numerical analyses and analytical models are pushing towards further investigations. 
In the present work, laser-assisted bending of stainless steel sheets to achieve sharp angles with limited fillet radii is therefore investigated. First, bending trials were performed by combining laser irradiation by a high power diode laser $(940 \pm 20 \mathrm{~nm}$, wavelength) with an auxiliary bending device based on kinematic of deformable quadrilaterals and triggered by a pneumatic actuator. Second, laser operational parameters, that is, scanning speed, power and number of passes, were investigated to identify the most suitable processing settings. In particular, the operational parameters were chosen to ensure bending angles close to the stroke limit imposed by the auxiliary bending device as well as the lowest fillet radii of the bent side of the workpiece. In this respect, bending angles and fillet radii were measured by coordinate measurement machine. Experimental data were elaborated by combined ANalysis Of Mean (ANOM) and ANalysis Of VAriance (ANOVA). Based on the experimental findings, the best strategy to achieve an aircraft from a stainless steel sheet was, lastly, designed and implemented, thus stating the suitability of laser-assisted bending to processing of complex shaped workpiece.

\section{Experimental}

\section{Materials and Equipments}

AISI 304 stainless steel sheet, $1 \mathrm{~mm}$ thick, was cut in blanks $50 \times 70 \mathrm{~mm}^{2}$. Prior to laser forming, the sheets surface was accurately cleaned by washing in ultrasonic bath of an ethanol solution.

Laser treatment was performed by a high power diode laser source (ROFIN-SINAR, DL 015, Hamburg, Germany) with a beam wavelength of $940 \mathrm{~nm} \pm 10 \mathrm{~nm}$. During laser processing, argon was flushed on the workpiece to minimize oxidation phenomena. The beam profile of a high power diode laser shows typically a rectangular shape with a top hat profile in one direction (slow axis) and a Gaussian profile in the other axis (fast axis). If the intersection of the beam profile with the focal plane is considered, this will result in the formation of an ellipse. The ellipse features in the focal plane a fast axis (i.e., parallel to the direction of the laser scanning pattern) of $1.2 \mathrm{~mm}$ and a slow axis of $3.8 \mathrm{~mm}$. Since emission from a single diode laser is well known to be confined to the narrow junction region $(\sim 1-2 \mu \mathrm{m})$, diffraction of the light should result in a large beam divergence of $\sim 35-45^{\circ}$ half angle in the direction perpendicular to the emission line ('Gaussian' or fast axis) and $\sim 5-10^{\circ}$ half angle in the direction parallel to the emission line ('slow' axis). The focal distance of the lens is $63 \mathrm{~mm}$, while the working distance is $32 \mathrm{~mm}$.

Laser assisted bending was performed by clamping the workpiece through an ad hoc designed device (Fig. 1). The bending device was in turn locked on a 1-axis CNC movement system to laser scan the prescribed bending pattern over the workpiece surface at constant and monitored speed. Figure 2 summarizes the kinematic of the device, which is based on a deformable quadrilateral with the top sides measuring $30 \mathrm{~mm}$ (white thick line) and a pneumatic piston. The system is thus triggered by a pneumatic actuator, which activates an extending/retracting piston, whose dynamic push is converted through the geometry of the deformable quadrilateral in a bending torque acting on the bending axis (Fig. 2). The geometry of the deformable quadrilateral is also designed to ensure the bending axis is always still during experiments, thus allowing it to keep the laser beam in focus over the bending direction (i.e., the scanning 


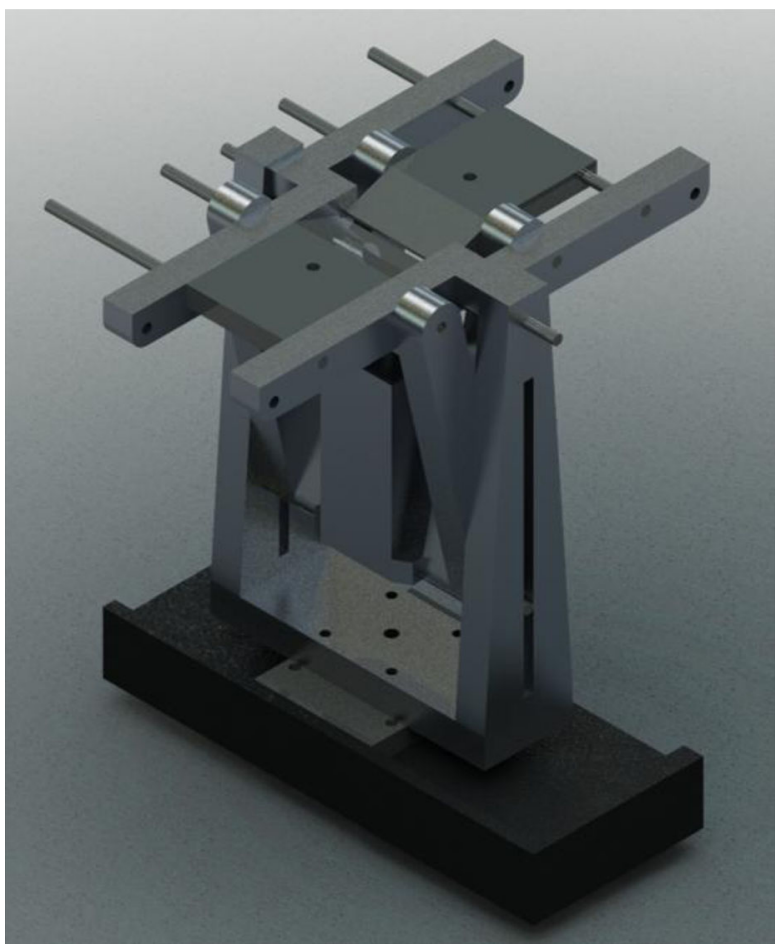

Fig. $13 \mathrm{~d}$ sketch of the bending device

pattern falls in the middle of the $50 \times 5 \mathrm{~mm}^{2}$ wide green area belonging to the workpiece surface in Fig. 3). The gap width was taken wide enough $(5 \mathrm{~mm})$ to safely allocate the major axis of the elliptical laser spot of $3.8 \mathrm{~mm}$. In this way, the bending zone is always irradiated by the laser beam at the scheduled power, this being the source of the thermal contribution to the bending torque exerted by the pneumatic piston. The clamping device also encompasses the locking system of the workpiece during laser assisted bending. Locking of the workpiece is ensured through two red rawplugs (Fig. 4), which lock the blank to the grey backings and allow the transmission of the bending torque.

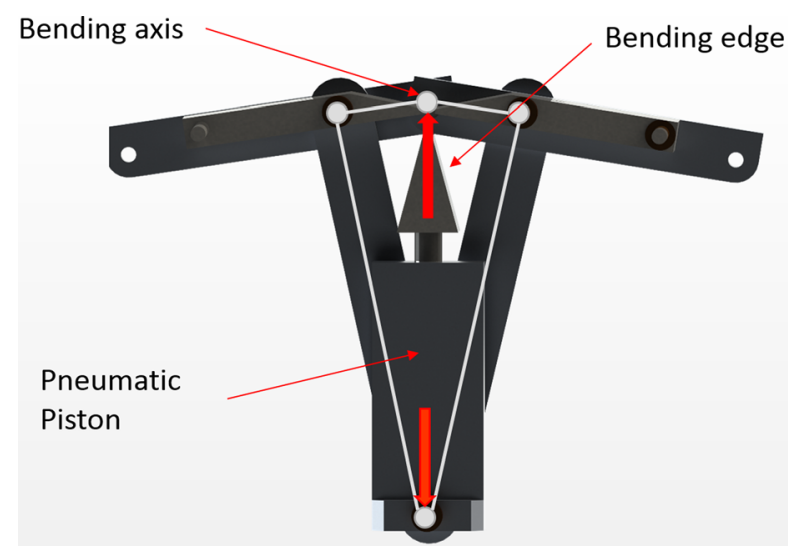

Fig. 2 Kinematic of deformable quadrilateral, pneumatic piston, bending axis and bending edge 


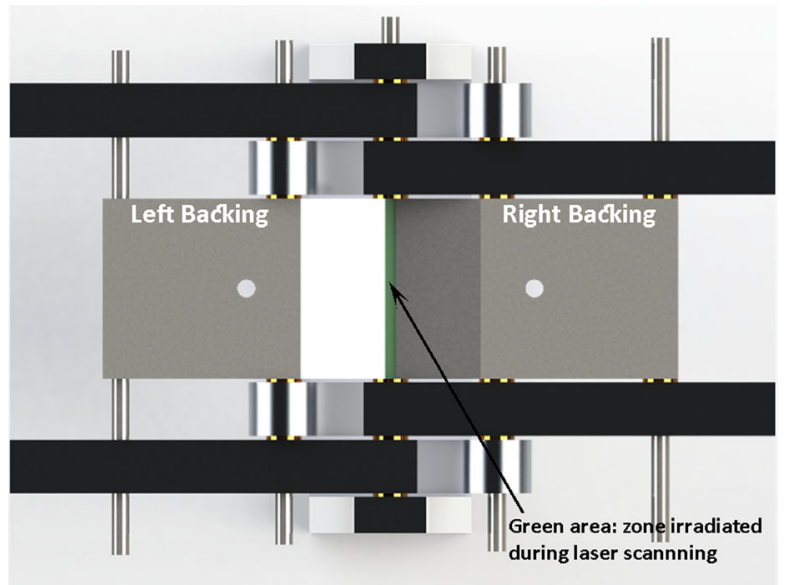

Fig. 3 Top view of the bending device, including the $50 \mathrm{~mm}$ long bending pattern

The commercially available piston works with compressed air in a range of 1 to 10 bar. It features an aluminium frame, a rod diameter of $20 \mathrm{~mm}$, a retracting force of $137 \mathrm{~N}$ and an extending force of $164 \mathrm{~N}$ at 6 bar. The maximum stroke of the piston is $300 \mathrm{~mm}$.

\section{Methods}

Bending experiments involve the following procedure: (i) measurement of the blank and tracing of reference geometry to hold the workpiece on the bending device; (ii) additional cleaning of the workpiece by solvent to eliminate any contaminants, which could modify the absorbance of the laser beam; (iii) positioning of the workpiece on the bending device; (iv) switching on air compressor at a constant pressure of 8 bar; (v) focusing of laser beam on the workpiece surface and setting of operational parameters; (vi) lining-up of the bending pattern and prescribed laser scanning direction; (vii) safety control and run of the experimental test; (viii) disarming the experimental apparatus; (ix) cooling-off the experimental apparatus and processed workpiece; (x) unlocking of the workpiece for characterization tests.

Table 1 summarizes the setting of the laser operational parameters, that is, scanning speed, laser power and number of passes. A full factorial experimental plan was developed. The experimental factors "laser power" and "number of passes" were varied over three experimental levels. The experimental factor "scan speed" was varied over

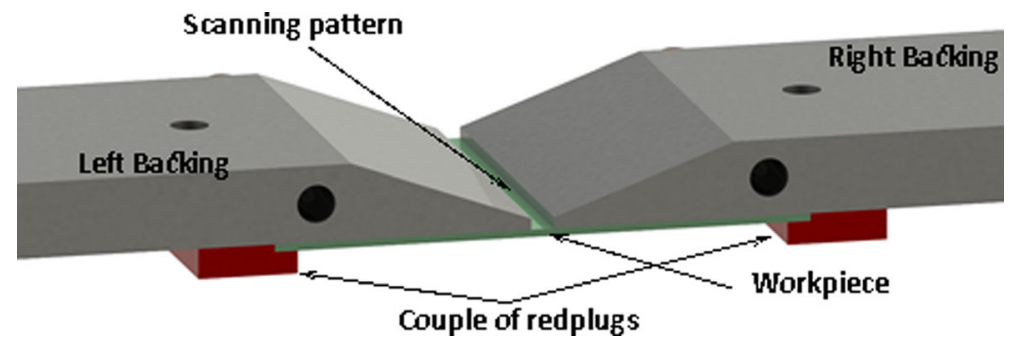

Fig. 4 Locking of the workpiece on the bending device 
two levels. A total number of 18 tests, replicated two times (36 tests) was performed. Main effects and interaction plots were built on to depict the trends of the experimental output, bending angle and filler radius (as better explained in the next section), according to the operational parameters. A main effects plot is a plot of the means of the experimental output at each level of an experimental factor. A main effect occurs when the mean response of the experimental output changes across the levels of an experimental factor. Accordingly, main effects plots can be used to compare the relative strength of the effects across factors on the experimental output. An interactions plot is a plot of means of the experimental output for each level of an experimental factor with the level of a second experimental factor held constant. Interactions plots are useful for judging the presence of interactions among experimental factors on the experimental output. Interaction is present when the response of the experimental output at a factor level depends upon the level(s) of other experimental factors. Parallel lines in an interactions plot indicate no interaction. The greater the departure of the lines from the parallel state, the higher the degree of interaction. Analysis of variance (ANOVA, confidence interval of 0.05) was also implemented for the statistical elaboration of the experimental data. Analysis Of VAriance (ANOVA) was used to analyze the available results and establish the meaningfulness of the operational parameters and of their experimental levels on the experimental output.

Preliminary tests allowed to identify the most promising setting of the operational parameters. When combination of laser power and scanning speed was taken outside of the prescribed range (Table 1), the laser contribution to the bending process was not effective or too much effective to impair the workpiece surface with severe burnings or thermal alterations. All the experimental trials were performed starting on workpiece at ambient temperature. During laser processing, the dynamic push of the pneumatic piston was always held constant at $220 \mathrm{~N}$ corresponding to a pressure of 8 bar, which is lower than the experimental load of approximately $326 \mathrm{~N}$ (as measured by preliminary tests on a static bending machine (MTS Insight 5, Eden Prairie, Minnesota, USA) on the metal workpiece), necessary for the onset of deformation in the plastic field of the workpiece. Accordingly, the mechanical push of the piston alone could not be sufficient to induce plastic deformation in the workpiece in the absence of thermal contribution of the laser irradiation. However, when the workpiece was heated by the multiple laser passes, its yield strength could progressively decrease and the bending torque exerted by the pneumatic piston was sufficient to cause the bending of metal to high angles. To manage the subsequent laser passes, the 1-axis CNC movement system drove the laser head along the prescribed pattern. The pattern was $60 \mathrm{~mm}$ long, that is $10 \mathrm{~mm}$ more than the workpiece width. The laser spot started its prescribed pattern $5 \mathrm{~mm}$ before the closest workpiece edge. Then, it moved through the workpiece,

Table 1 Setting of laser operational parameters

\begin{tabular}{lrrr}
\hline Experimental factor & Experimental levels & & \\
\hline Laser power $(\mathrm{W})$ & 125 & 150 & 175 \\
Scan speed $(\mathrm{mm} / \mathrm{s})$ & 1,5 & 2 & - \\
$\mathrm{N}^{\circ}$ of passes & 9 & 12 & 15 \\
\hline
\end{tabular}


stopping $5 \mathrm{~mm}$ after the farthest (opposite) workpiece edge. After that, the motion of the laser head was inverted, driving the laser spot along its way back to the starting point. This process was repeated for each laser pass. The tests were replicated two times for ensuring replicability and repeatability of the experimental results.

\section{Measurements}

Dimensional measurements of the workpiece was performed by a coordinate measuring machine (DEA HEXAGON Global Classic 05.05.05, Cobham, United Kingdom). The shape of the workpiece was measured by storing 11 profiles equally spaced over the surface with a resolution of $1.25 \mu \mathrm{m}$ (Fig. 5). Each profile corresponds to a bending profile. The bending profile is composed of three segments: two linear branches and a bend at the top of the profile. It provides a measure of the fillet radius (i.e., the radius of curvature of the u-bend at the top of the profile) and bending angle (i.e., the angle formed by crossing the two linear branches of the bending profile). The fillet radius and bending angle were calculated by averaging the experimental data of the 11 profiles stored per each workpiece. However, the samples, failing to achieve acceptable surface status after laser assisted mechanical bending, were disregarded. The surface was considered severely impaired in the case of visible local deformation generated by the laser, that is, local distortion of the workpiece edges, local melting of the metal, formation of holes or accumulation of molten materials sideways the laser scanning patterns.

\section{Results and Discussion}

\section{Analysis of the Bending Mechanisms}

The bending of the metal blanks are achieved by combination of the mechanical push of the pneumatic piston (bending torque) and the thermal contribution of the laser beam, which decreases the yield strength of the metal being bent. Therefore, the final shape of

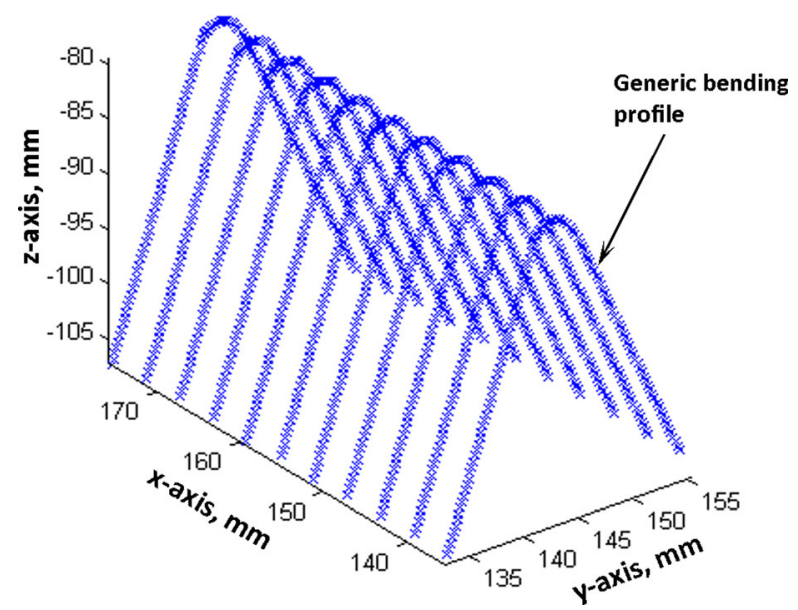

Fig. 5 Storing of profiles by coordinate measurement machine after workpiece bending 
the bent workpiece follows the profile of the bending edge in Fig. 2, thus giving rise to the silhouette reported in Fig. 5. The orientation of the silhouette is not dependent on the contribution of the laser processing (especially, of the bending mechanism by the thermal contribution). It depends on the kinematic of the clamping device (Fig. 2) and, especially, on the bending torque the pneumatic piston exerts on the workpiece. For this reason, the workpiece is bent opposite to the direction of the laser irradiation.

Figure 6 reports the trends of the bending angle vs. the laser operational parameters, that is, laser power, scanning speed and number of passes. Laser assisted bending allows the achievement of bending angles ranging from over 70 to $140^{\circ}$ (that is, the stroke limit imposed to the bending process by the auxiliary bending device and, especially, by the design of the bending edge). Figure 6 also shows the increasing bending angles when higher fluence or irradiance is set to process the workpiece surface. This means an increased number of passes, lower scanning speed and/or higher laser power allow bending angles close to the stroke limit. In particular, setting the laser power at $175 \mathrm{~W}$ and number of passes at 15 , both the scanning speeds of 1.5 and $2.0 \mathrm{~mm} / \mathrm{s}$ allow getting the maximum bending angle. When the number of passes is reduced to 12 , the only combination, which allows reaching the maximum bending angle, is the highest power of $175 \mathrm{~W}$ with the lowest scanning speed of $1.5 \mathrm{~mm} / \mathrm{s}$. Reducing the number of passes to 9 means the thermal contribution by the laser beam is not sufficient to lead to the maximum bending angles.

Bending angles of 70 to $140^{\circ}$ shown in Fig. 6 are very high and, as mentioned, they can be attributed to the superimposition of an external load (i.e., the push of the pneumatic piston in the elastic field) with the laser beam irradiation. During the bending process of the metal blanks, the laser beam irradiates the workpiece and generates a localized temperature increase and, accordingly, a progressive decrease in the mechanical strength of the metal. In this way, the push of the pneumatic piston becomes effective to deform permanently the workpiece. Laser contribution to the establishment of the final shape of the workpiece is ascribed to the thermal effect on
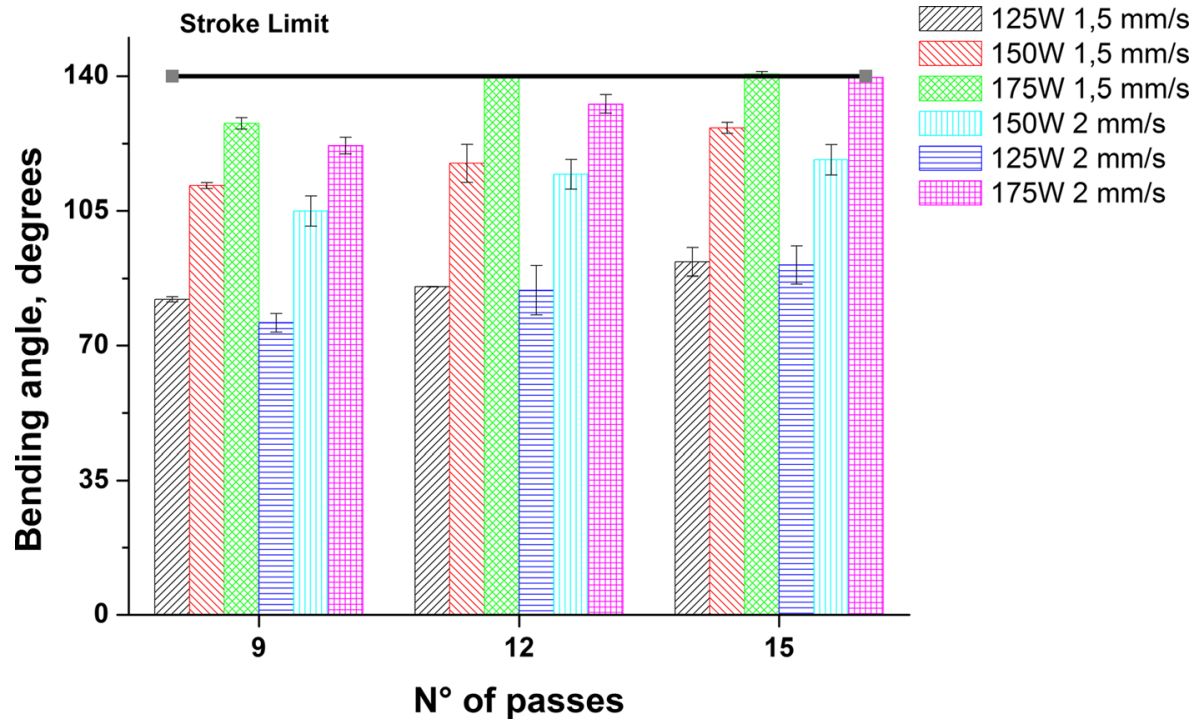

Fig. 6 Bending angles vs. laser operational parameters 
the metal according to the Buckling Mechanism (BM). As known, BM occurs at low scan speed of the laser beam and moderate power density as this is the case, when temperature is rather uniform across the thickness of the workpiece. Moreover, being the size of the laser beam bigger than the workpiece thickness, BM is favored. In the presence of BM, the material is usually formed towards the same side of the irradiation [3, 9]. However, when an external source as the action of an external bending torque acts on the workpiece, the external force essentially drives the orientation of the workpiece. High bending angles in laser assisted forming of metal blanks is not unlikely to occur. Roohi et al. [19] studied an external force-assisted laser bending of metallic and non metallic sheets through cantilever clamping arrangement and multiple scanning passes. However, their target was the reach of bending angles of $90^{\circ}$, which is by far less than the nominal value of $140^{\circ}$ herein investigated. Similarly, Magee and De Vin [16] reported a process planning for laserassisted forming of metal blanks at $90^{\circ}$ bending angle. They found a strict relation between the shaping of metal blanks to high angles and the interaction between thermal and mechanical contributions.

In order to better understand the role of thermal and mechanical contribution, Che Jamil et al. [3] report bending angles of only 1 to $8^{\circ}$ by laser bending according to $\mathrm{BM}$ on $0.9 \mathrm{~mm}$ thick AISI 304 blanks using fiber laser with circular beam of $16 \mathrm{~mm}$ in diameter and power from 400 to $1000 \mathrm{~W}$. Therefore, the obtuse bending angles (in the range of 70 to $140^{\circ}$ ) herein reported can be only explained in the light of combined thermal and mechanical effects during laser-assisted bending, which boosts the achieved bending angles. In particular, heating by laser source can be inferred to modify the microstructure of the stainless steel blanks in agreement with the experimental findings in [24]. Heating is known to promote the establishment of bigger crystallite in the metal, thus increasing the suitability of the metal microstructure to be plastically deformed and reducing the flexural torque necessary for the achievement of large deformation [10]. In agreement with the previous considerations, the mechanical push of $220 \mathrm{~N}$, which, at ambient temperature, is found to be not sufficient to plastically deform the stainless steel blanks, becomes sufficient to support the bending operation during the laser assistance and leads, in the present work, to obtuse bending angles. In the literature, Geiger et al. [5] shows an increase in the effectiveness of the bending process of up to $200 \%$, when laser beam and mechanical bending are superimposed. This result is attributed by Geiger et al. to a sort of thermal annealing of the steel microstructure, which increases metal suitability to deformation, despite the very low interaction time between laser source and metal should prevent any form of annealing.

Statistical Analysis of the Experimental Results

Figures 7 and 8 report the plot of Main Effects Plot (MEP) and the Interactions Plot (IP) for the experimental output "bending angle". Table 2 reports the ANalysis Of VAriance (ANOVA). The trends of the bending angle in ANOM plot confirms the previous considerations about the influence of an increase in the power density delivered to the workpiece on the final shape achievable. The trends of bending angles in IP show the 
lacking of any significant interaction among the experimental factors, as they run approximately parallel. ANOVA table shows Fisher's factors for laser power, number of passes and scanning speed and their interactions. Each experimental factor contributes significantly to the variations of the experimental output "bending angle", with laser power being the most contributing one ( $\mathrm{F}$ of approximately 728 ). As said before, interactions among factors do not contribute significantly to the variations of the experimental output, with Fisher's factors being low (approximately 1 or less) and much lower than the corresponding tabulated ones.

Figure 9 reports the trends of the fillet radius vs. the laser operational parameters, that is, laser power, scanning speed and number of passes. The fillet radius is found to decrease when higher power density is irradiated on the workpiece surface. This means an increased number of passes, lower scanning speed and/or higher laser power allows the establishment of the lowest fillet radii. Accordingly, the sharpest angles with the smallest fillet radii are achieved together with the largest bending angle. It is therefore possible to achieve very narrow fillet radii of less than $2 \mathrm{~mm}$ for bending angles close to
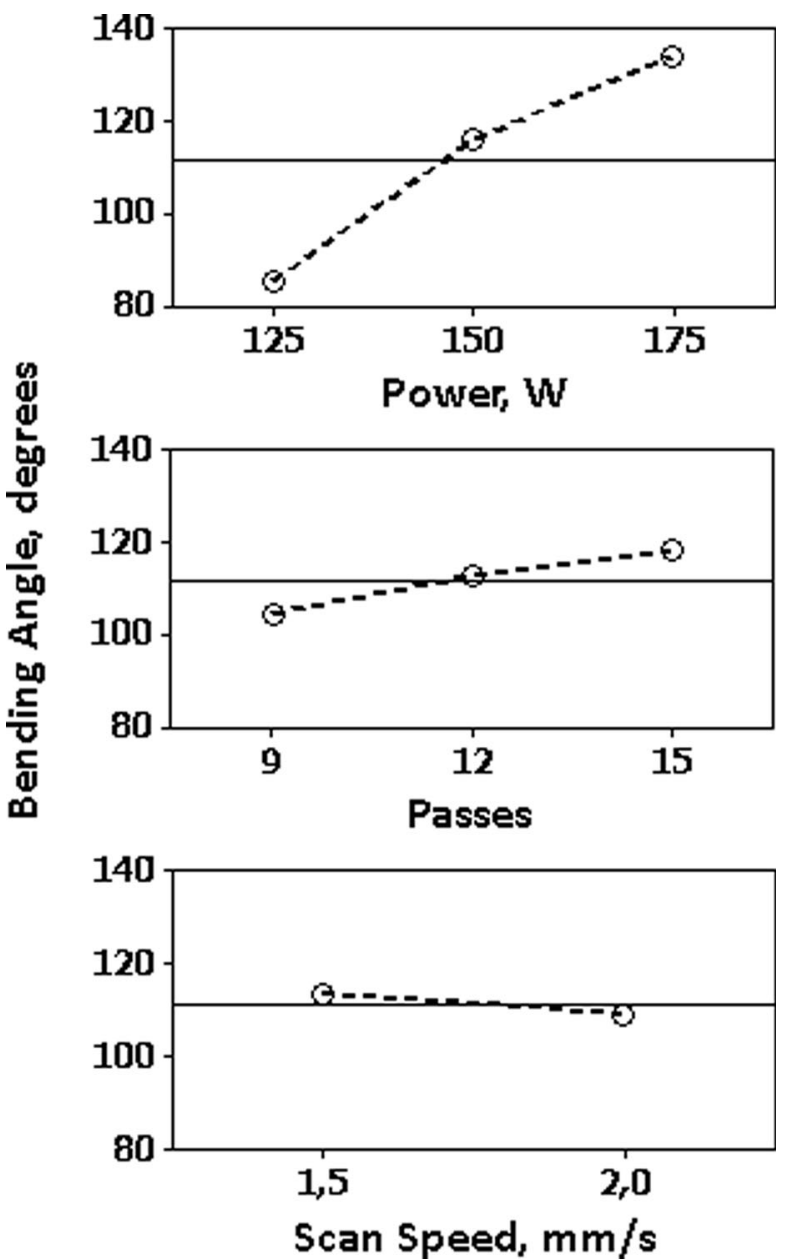

Fig. 7 ANOM plot of bending angles vs. laser operational parameters 


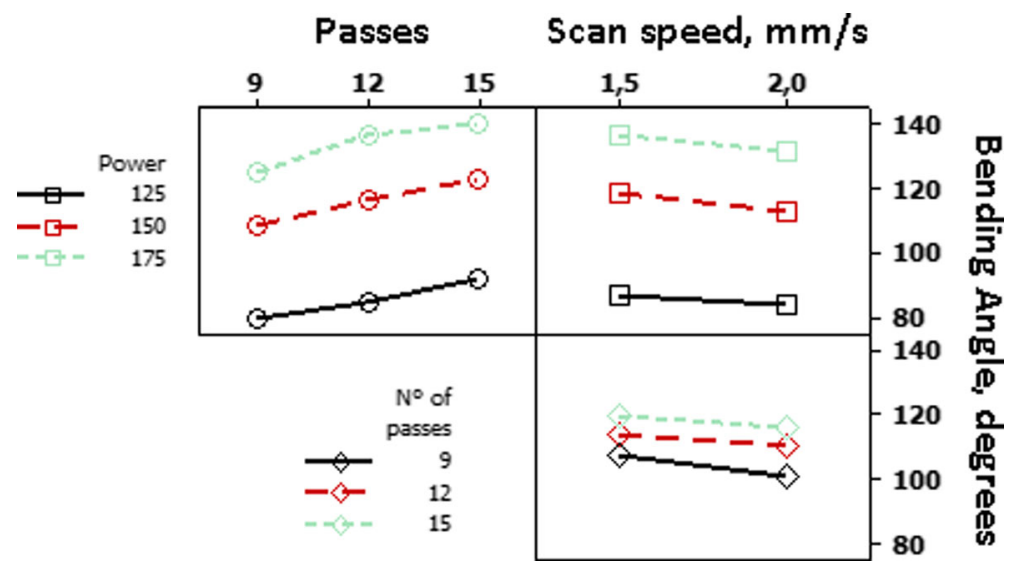

Fig. 8 Interactions plot of bending angles vs. laser operational parameters

the stroke limit, which is very unlikely to occur by mechanical shaping as, for example, shown by [22].

Figures 10 and 11 report the plot of Main Effects Plot (MEP) and the Interactions Plot (IP) for the experimental output "fillet radius". Table 3 reports the ANalysis Of VAriance (ANOVA). The trends of the fillet radius in MEP plot confirms the previous considerations about the influence of power density increase on the final shape of the workpiece. The trends of the fillet radii in IP shows some interactions among the experimental factors, especially for the factor "laser power" with the factor "number of passes", which influence the experimental output "fillet radius". The interactions among the experimental factors could mostly influence the experimental output "fillet radii" at high and moderate level of laser power. In this case, the setting of the other two parameters (number of passes, and, at a minor extent, scanning speed) can be discriminant to the level of irradiance/fluence delivered to the workpiece and, therefore, to the final shape achievable.

Table 2 ANOVA table of bending angles vs. laser operational parameters. DoF stands for Degree of Freedom, SS stands for Sum of Squares, MS for Mean Square (SS/DoF), F is the Fisher's factor and P is the percentage of contribution

\begin{tabular}{lcrrrr}
\hline Source & DoF & \multicolumn{1}{c}{ Adj SS } & Adj MS & \multicolumn{1}{l}{$F$} & \multicolumn{1}{l}{$P$} \\
\hline Power & 2 & 14606.07 & 7303.03 & 727.98 & 0.000 \\
Passes & 2 & 1174.73 & 587.37 & 58.55 & 0.000 \\
Speed & 1 & 178.20 & 178.20 & 17.76 & 0.001 \\
Power*Passes & 4 & 37.72 & 9,43 & 0.94 & 0.463 \\
Power*Speed & 2 & 15.11 & 7.55 & 0.75 & 0.485 \\
Passes*Speed & 2 & 13.52 & 6.76 & 0.67 & 0.522 \\
Power*Passes*Speed & 4 & 41.37 & 10.34 & 1.03 & 0.418 \\
Error & 18 & 180.58 & 10.03 & & \\
Total & 35 & 16247.28 & & &
\end{tabular}




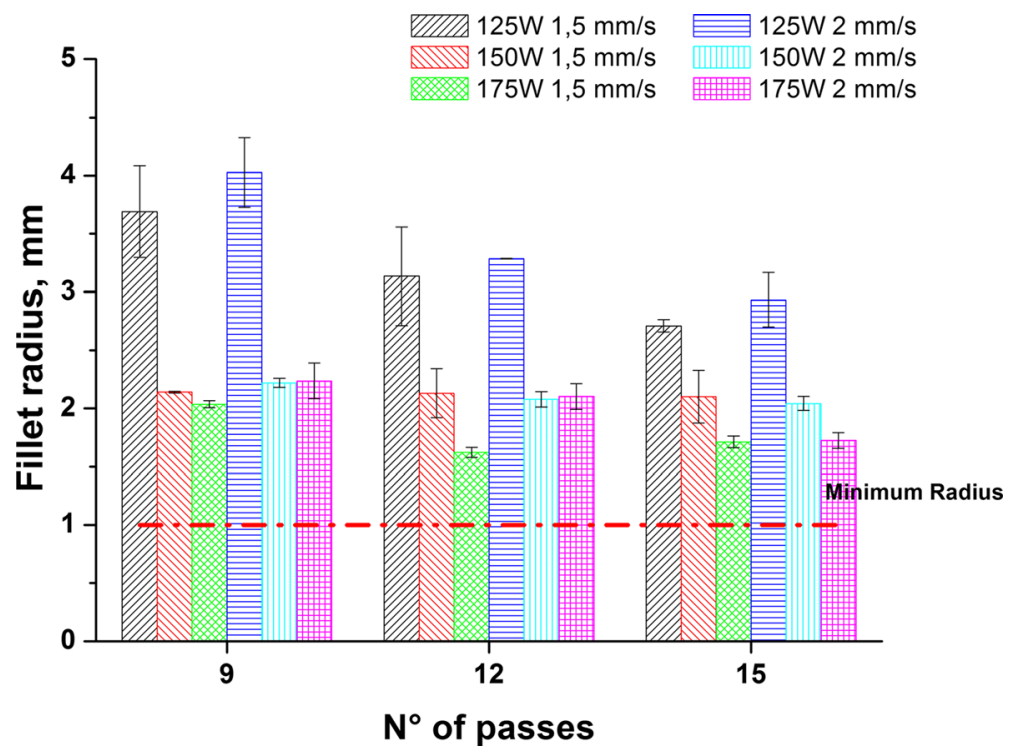

Fig. 9 Fillet radii vs. laser operational parameters

Fillet radii is strictly dependent on the geometrical features of the thermally altered zone. At high power, thermally altered zone can be coarse as a result of the partial melting or local overheating of the irradiated surface, which can affect the surface morphology of the workpiece. Under these process conditions, upset mechanism (UM) can take place together with buckling mechanism (BM) in agreement with the experimental findings in [8]. Concurrent forming by UM and BM can affect the accuracy and size of the fillet radii. In this case, the scan speed of the laser beam is very low and power density moderate. These settings cause temperatures to be rather uniform across the thickness of the workpiece. In agreement with [11], when temperature gradient in the material is moderate and the size of the laser spot is closer to workpiece thickness, UM can also occur, thus causing the thickening of the irradiated portion of the material and bending by instability (i.e., out-of-plane bending). In contrast, when the laser power is set at a low level, the interactions with the other operational parameters is of minor importance. ANOVA table shows Fisher's factors for laser power, number of passes and scanning speed and their interactions. Each experimental factor contributes to the experimental output "fillet radius", with laser power being by far the most contributing one. The factor "scanning speed" is apparently less influential on the experimental output "fillet radius", this being also ascribable to the small experimental range investigated. As said before, the interaction between the experimental factors "laser power" and "scanning speed" can contribute to the experimental output "fillet radius", with Fisher's factor being higher or, however, close to the corresponding tabulated one.

\section{Manufacture of An Aircraft Prototype}

Based on the experimental results retrieved in the first part of this work, some trials to manufacture an aircraft prototype by bending of appropriate steel blanks (Fig. 12) are 

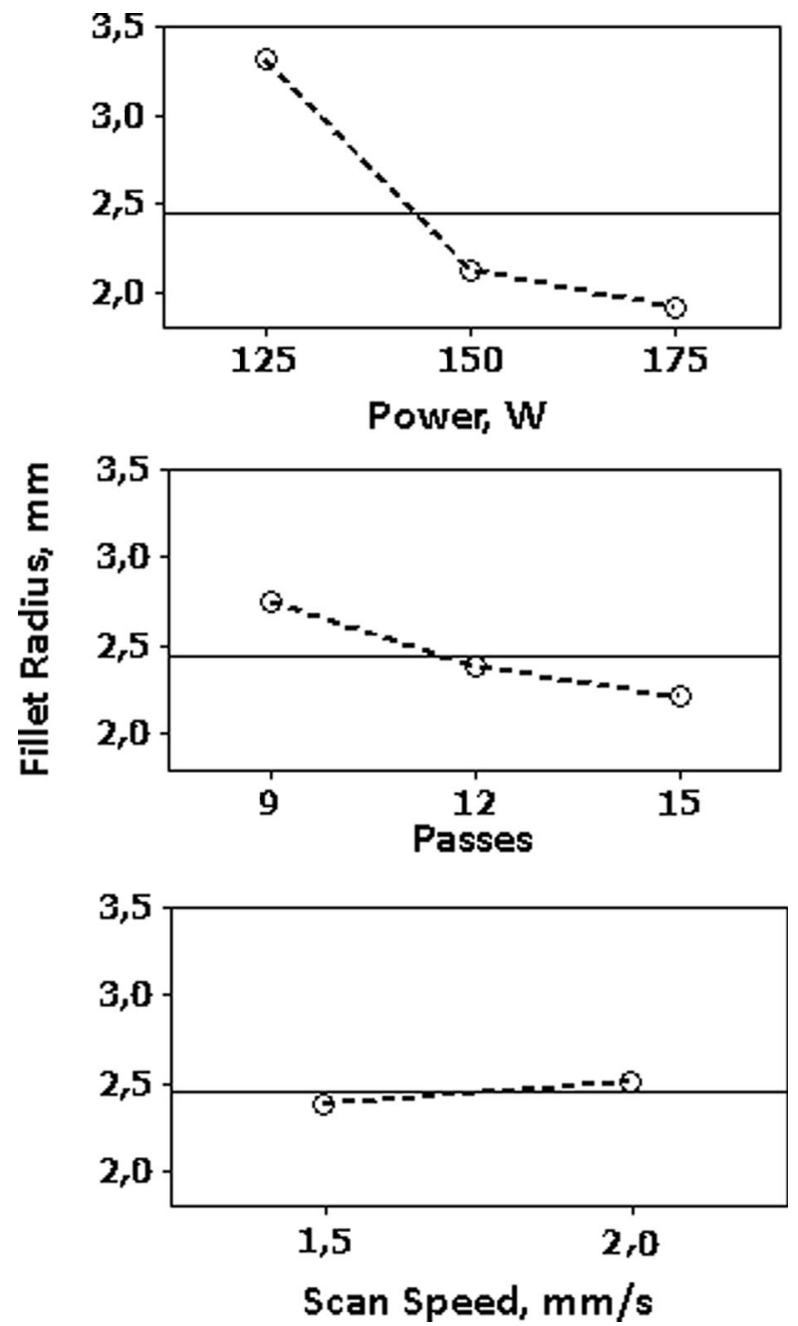

Fig. 10 ANOM plot of fillet radii vs. laser operational parameters

developed. First, bending process is operated on the central line of the stainless steel blank in Fig. 12i by laser-assisted bending (i.e., using the same auxiliary device before described) according to BM. For this purpose, scanning speed is kept low at $1.5 \mathrm{~mm} / \mathrm{s}$ and number of passes set at 15 , respectively. The settings of the operational parameters are suggested by the previous experimental analysis. The scan speed of the laser beam is set at the lowest level of $1.5 \mathrm{~mm} / \mathrm{s}$, while the high number of passes is set at the highest level of 15. In agreement with the experimental results reported in Figs. 6 and 9, this choice should allow achieving the smallest fillet radii (maximum accuracy of the bends) and highest bending angles (i.e., close to the target values imposed by the stroke limit of the auxiliary bending device). For practical purposes, the bending line (i.e., the laser scanning pattern) is split in three segments of 4, 42 and $8 \mathrm{~mm}$, where laser power is set at 100, 155 and $60 \mathrm{~W}$, respectively. Only the intermediate section is irradiated at the full power to avoid thermal alteration of the workpiece edges. As known, the outer 


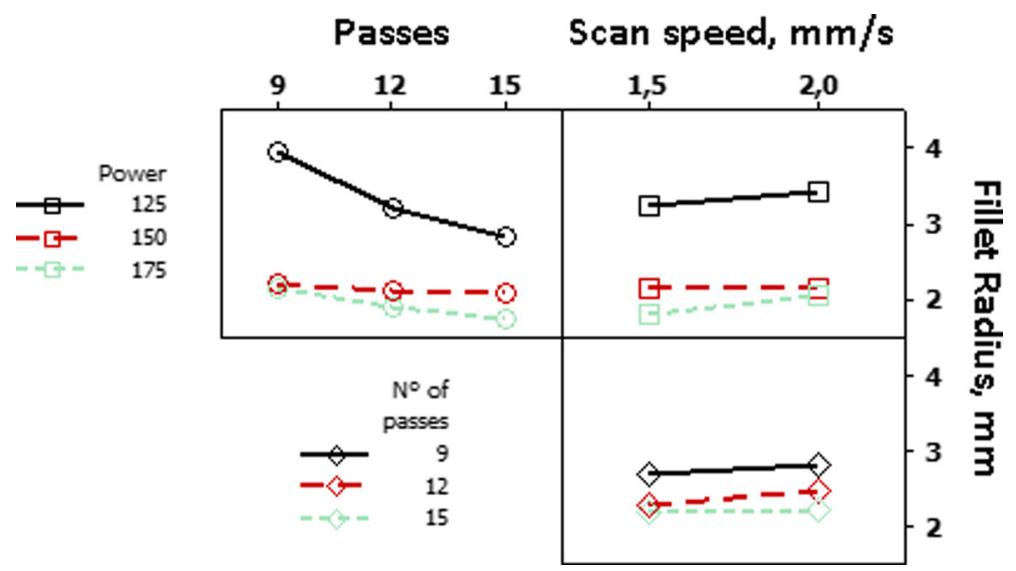

Fig. 11 Interactions plot of fillet radii vs. laser operational parameters

portion of the workpiece is much more susceptible to overheating during laser processing, being it unable to exchange effectively the heat on all the sides. The overheating of the edges can lead to the workpiece distortion and, therefore, it should be carefully avoided. The first segment is irradiated by the laser beam at $100 \mathrm{~W}$, as the workpiece starts at ambient temperature. The last segment is irradiated at only $60 \mathrm{~W}$, to compensate the pre-heating of the workpiece by the laser source during the first two segments. The choice of the lengths of the aforementioned scanning segments is based on practical issues. Longer "safe zones" at the edges of the workpiece would be better to preserve the material integrity, but they would increase abnormally the manufacturing time. Accordingly, the "safer zones" at the edges of the workpiece of 4 and $8 \mathrm{~mm}$ are set as good compromise between the need for safeguarding the metal workpiece and acceptable processing time.

First bending operation performed by the irradiation of the central line of the workpiece depicted in Fig. 12i allows achieving a large bending angle of approximately

Table 3 ANOVA table of fillet radii vs. laser operational parameters. DoF stands for Degree of Freedom, SS stands for Sum of Squares, MS for Mean Square (SS/DoF), F is the Fisher's factor and P is the percentage of contribution

\begin{tabular}{lcclrl}
\hline Source & DoF & Adj SS & Adj MS & \multicolumn{1}{l}{$F$} & \multicolumn{1}{l}{$P$} \\
\hline Power & 2 & 13.78 & 6.89 & 167.74 & 0.000 \\
Passes & 2 & 1.87 & 0.93 & 22.71 & 0.000 \\
Speed & 1 & 0.15 & 0.15 & 3.59 & 0.074 \\
Power*Passes & 4 & 1.11 & 0.28 & 6.75 & 0.002 \\
Power*Speed & 2 & 0.09 & 0.05 & 1.13 & 0.344 \\
Passes*Speed & 2 & 0.02 & 0.01 & 0.26 & 0.770 \\
Power*Passes*Speed & 4 & 0.11 & 0.03 & 0.68 & 0.612 \\
Error & 18 & 0.74 & 0.04 & & \\
Total & 35 & 17.86 & & & \\
\end{tabular}


(i)

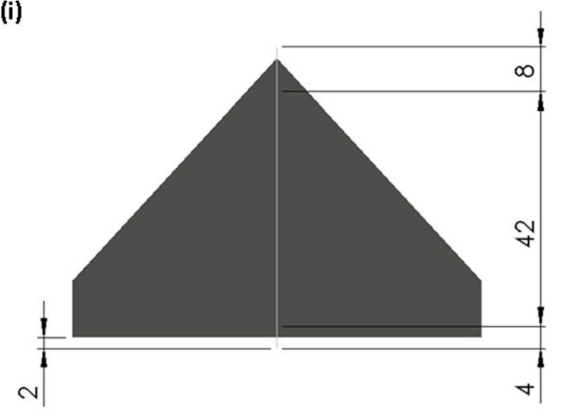



(iv)

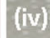

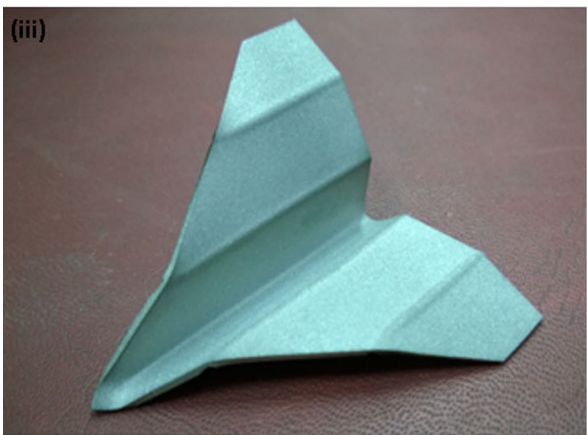

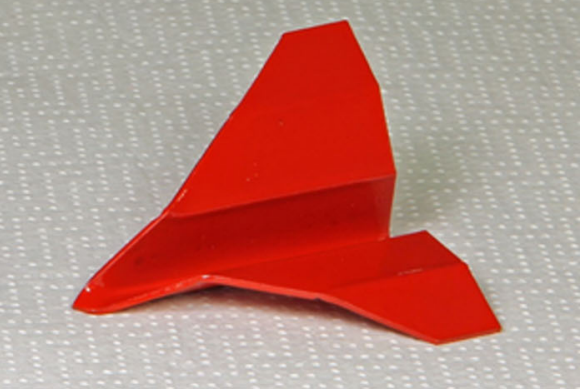

Fig. 12 Bending of an aircraft from steel blanks: (i) design of bending strategy for the central line; (ii) design of bending strategy for the lateral bending; (iii) raw aircraft after bending; (iv) painted aircraft

$135^{\circ}$, with a rather small filler radius of $1.93 \mathrm{~mm}$. This result are in good agreement with experimental data reported in Figs. 6 and 9.

After bending of the central line of the stainless steel blank, additional forming of the wings are performed along two lines located at 12 and $24 \mathrm{~mm}$ from the left and right rims of the metal blank (Fig. 12ii) by laser bending according to the Thermal Gradient Mechanism (TGM) [11]. Wings bending by laser according to TGM do not rely on previous experimental results. However, they demonstrate how the combination of BM and hybrid thermos-mechanical bending with TGM and laser bending could allow the achievement of the peculiar aircraft shapes shown in Fig. 12. According to TGM, the side of the workpiece directly irradiated by laser is rapidly heated and approached a higher temperature than the opposite side, thus expanding more. Tensile stress is generated inside the layers of material directly exposed to laser source, while, on the other side, compressive stress arises. On cooling off, the irradiated side contracts more and the workpiece bends toward the laser source because of the inversion of the residual stress fields, with the topmost layer being in compression and the down most in traction. For this purpose, the laser power is set at an higher $200 \mathrm{~W}$, the number of passes at 12 , while the scan speed of the laser spot is increased at $6 \mathrm{~mm} / \mathrm{s}$. Being, in this case, the scanning speed much higher, TGM takes place. No particular cautions are taken to modulate the laser power along the scanning pattern to avoid thermal distortion of the metal blank, being the interaction time between the laser source and workpiece definitely lower. Figures $12 \mathrm{iii}$ and $12 \mathrm{iv}$ show the final shape of the aircraft after the pre-treatment before the painting process and after the painting process. The lines 
located at 12 and $24 \mathrm{~mm}$ from the left and right rims of the metal blank are bent forming angles of approximately 45 and $22.5^{\circ}$ respect to the direction of the sides bent by laser-assisted bending around the central line of the workpiece. The prescribed shapes are achieved with a good level of accuracy and precision. Minimal thermal alterations of the blank can be deduced by the good visual appearance of the aircraft after painting. This result demonstrates the suitability of the laser-assisted bending in forming complex shapes or, at least, playing a crucial role in troublesome shaping operations of metal workpiece.

\section{Conclusions}

In the present work, laser-assisted bending by external forces of stainless steel sheets to achieve sharp angles is experimentally investigated. The following results can be drawn:

- Large bending angle of 70 to $140^{\circ}$ are achieved by laser assisted bending by external forces. The shape of the workpiece after bending is the result of the Buckling Mechanism (BM) and the shaping action of the bending torque exerted by the auxiliary forming device.

- Large bending angles can be interpreted in the light of the superimposition of the thermal and mechanical forming of the workpiece, with laser thermal heating being able to make the stainless steel more prone to bending and mechanical push more effective.

- The bending angle is found to increase when higher fluence or irradiance is used to process the workpiece surface. This means an increased number of passes, lower scanning speed and higher laser power allows bending angles close to the stroke limit of $140^{\circ}$.

- The fillet radius is found to decrease when higher fluence or irradiance is used to process the workpiece surface. This means an increased number of passes, lower scanning speed and higher laser power allows the establishment of the lowest fillet radii. Accordingly, the sharpest angles with the smallest fillet radii of less than $2 \mathrm{~mm}$ are achieved together with the largest bending angle of $140^{\circ}$. It is therefore possible to achieve very narrow fillet radii for bending angles close to the nominal value of $140^{\circ}$, which is very unlikely to occur after mechanical shaping.

- Fillet radii is found to be strictly dependent on the features of the thermally altered zone. At high power, thermally altered zone can be very coarse as a result of the thermal alteration of the metal. In these process conditions, upset mechanism (UM) can take place together with buckling mechanism (BM) and this can affect the accuracy and size of the fillet radii.

Based on experimental findings, the best strategy to achieve an aircraft from a stainless steel sheet through the combination of laser assisted bending by buckling and thermal gradient mechanism was designed and implemented. The prescribed shapes were achieved with a good level of accuracy and precision. Minimal thermal alterations can be deduced by the good visual appearance of the aircraft after painting. 


\section{References}

1. Barletta, M., Casamichele, L., Tagliaferri, V.: Line bending of $\mathrm{Al}_{2} \mathrm{O}_{3}$ coated and uncoated aluminium thin sheets. Surf. Coat. Technol. 201, 660-673 (2006)

2. Carden, W.D., Geng, L.M., Matlock, D.K., Wagoner, R.H.: Measurement of springback. Int. J. Mech. Sci. 44, 79-101 (2002)

3. Che Jamil, M.S., Sheikh, M.A., Li, L.: A study of the effect of laser beam geometries on laser bending of sheet metal by buckling mechanism. Opt. Laser Technol. 43, 183-193 (2011)

4. Cho, J.R., Moon, S.J., Moon, Y.H., Kang, S.S.: Finite element investigation on spring-back characteristics in sheet metal U-bending process. J. Mater. Process. Technol. 141, 109-116 (2003)

5. Geiger, M., Merklein, M., Pitz, M.: Laser and forming technology - an idea and the way of implementation. J. Mater. Process. Technol. 151, 3-11 (2004)

6. Geng, L.M., Wagoner, R.H.: Role of plastic anisotropy and its evolution on springback. Int. J. Mech. Sci. 44, 123-148 (2002)

7. Gisario, A., Barletta, M., Conti, C., Guarino, S.: Springback control in sheet metal bending by laserassisted bending: experimental analysis, empirical and neural network modelling. Opt. Lasers Eng. 49, $1372-1383$ (2011)

8. Hennige, T.: Development of irradiation strategies for 3D laser forming. J. Mater. Process. Technol. 103, $102-108$ (2000)

9. Hu, Z., Kovacevic, R., Labudovic, M.: Experimental and numerical modeling of buckling instability of laser sheet forming. Int. J. Mach. Tools Manuf. 42, 1427-1439 (2002)

10. Köhler, B., Bomas, H., Hunkel, M., Lütjens, J., Zoch, H.-W.: Yield strength behaviour of carbon steel microsheets after cold forming and after annealing. Scr. Mater. 62, 548-551 (2010)

11. Li, L.: The advances and characteristics of high power diode laser materials processing. Opt. Lasers Eng. 34, 231-253 (2000)

12. Lim, H., Lee, M.G., Sung, J.H., Kim, J.H., Wagoner, R.H.: Time-dependent springback of advanced high strength steels. Int. J. Plast. 29, 42-59 (2012)

13. Lan, F., Chen, J., Lin, J.: A method of constructing smooth tool surfaces for FE prediction of springback in sheet metal forming. J. Mater. Process. Technol. 177, 382-385 (2006)

14. Li, K.P., Carden, W.P., Wagoner, R.H.: Simulation of springback. Int. J. Mech. Sci. 44, 103-122 (2002)

15. Liu, Y.C.: The effect of restraining force on shape deviations in flanged channels. ASME J. Eng. Mater. Technol. 110, 389-394 (1988)

16. Magee, J., De Vin, L.J.: Process planning for laser-assisted forming. J. Mater. Process. Technol. 120, 322326 (2002)

17. Moon, Y.H., Kang, S.S., Cho, J.R., Kim, T.G.: Effect of tool temperature on the reduction of the springback of aluminum sheets. J. Mater. Process. Technol. 132, 365-368 (2003)

18. Papeleux, L., Ponthot, J.P.: Finite element simulation of springback in sheet metal forming. J. Mater. Process. Technol. 125-126, 785-791 (2002)

19. Roohi, A.H., Gollo, M.H., Naeini, H.M.: External force-assisted laser forming process for gaining high bending angles. J. Manuf. Process. 14, 269-276 (2012)

20. Sai, K.: Multi-mechanism models: present state and future trends. Int. J. Plast. 27, 250-281 (2011)

21. Schuöcker, D.: Mathematical modeling of laser-assisted deep drawing. J. Mater. Process. Technol. 115, 104-107 (2001)

22. Sun, J.S., Lee, K.H., Lee, H.P.: Effects of geometry and fillet radius on die stresses in stamping processes. J. Mater. Process. Technol. 104, 254-264 (2000)

23. Sunseri, M., Cao, J., Karafillis, A.P., Boyce, M.C.: Accommodation of Springback in channel forming using active binder control. ASME J. Eng. Mater. Technol. 118, 426-435 (1996)

24. Yilbas, B.S., Arif, A.F.M., Abdul Aleem, B.J.: Laser bending of AISI 304 steel sheets: thermal stress analysis. Opt. Laser Technol. 44, 303-309 (2012)

25. Zhao, J., Zhai, R., Qian, Z., Ma, R.: A study on springback of profile plane stretch-bending in the loading method of pretension and moment. Int. J. Mech. Sci. 75, 45-54 (2013)

26. Zhu, Y.X., Liu, Y.L., Yang, H., Li, H.P.: Development and application of the material constitutive model in springback prediction of cold-bending. Mater. Des. 42, 245-258 (2012) 